\title{
Microfluidic Single Mammalian Cell Lysis in Picolitre Droplets
}

\author{
Ramesh Ramji ${ }^{1}$, Ang Chee Xiang ${ }^{2}$, Ng Jia Ying ${ }^{1}$, Lim Chwee Teck ${ }^{1,2,3}$ and Chen Chia Hung ${ }^{1,4 *}$ \\ ${ }^{1}$ Department of Bioengineering, National University of Singapore, Singapore \\ ${ }^{2}$ Department of Mechanical Engineering, National University of Singapore, Singapore \\ ${ }^{3}$ Mechanobiology Institute, Singapore \\ ${ }^{4}$ Singapore Institute for Neurotechnology (SiNAPSE), Singapore
}

\begin{abstract}
One of the essential issues in cell biology is to analyze intracellular proteins that play an important role in signal transduction and processes. Standard assays for intracellular proteins are based on ensemble measurements which rule out the differences of intracellular protein expression in individual cells. Especially in cancer biology, only a few tumour cells turn into circulating tumour cells causing metastasis. In such cases, understanding the heterogeneity in cellular physiology would be necessary. To screen for intracellular protein expression among individual cells effectively, a reliable method to lyse individual cells in a high throughput manner is essential. Here, we demonstrate an integrated microfluidic platform combining a cell focusing channel, reagent injection inlet and a droplet generator for high throughput single cell lysis. Individual cells are encapsulated into the droplets with chemical reagents which result in disrupting the cell membrane integrity, causing cell lysis. High throughput mammalian cell lysis was performed thus facilitating intracellular protein analysis on a micro device.
\end{abstract}

\section{Introduction}

Bulk responses from a group of cells can often be misleading due to averaging of the signal from the subpopulation while that from individual cells carry more value since they could portray multimodal distribution among them. Intracellular heterogeneity among cells can be studied by monitoring their responses by means of high-throughput techniques [1]. However, most of these methods are limited by the availability of biomarkers that detect these compounds without lysing the cells [2]. For example, flow cytometry was initially optimized to screen the surface biomarkers in individual cells, but to measure the intra-cellular signals from individual cells remains challenging [3] since permeabilization of cells prior to the flow analysis could cause loss of intracellular proteins by diffusing out into the bulk phase.

Current methods of mammalian cell lysis include optical, mechanical, electric and chemical lysis techniques [4]. Optical lysis involves directing a $532 \mathrm{~nm}$ laser pulse at a designated cell [5]. The focused pulse creates a cavitation bubble on the cell which expands and disrupts the cells. The cells within the zone of injury are selectively lysed as a result of this method. Mechanical lysis makes use of sharp surfaces with nanoscale barbs termed 'nanoknives'. Cells are driven through a grating composed of serrated features, usually 2-3 nm apart, created using multiple isotropic deep reactive ion etch stages. This method causes $99 \%$ cell lysis according to a trypan blue quenching assay [6]. The electrical lysis technique generates transmembrane potentials of the order of $0.2-1.5 \mathrm{~V}$ that cause rupture of the lipid bilayer forming pores and depends on membrane composition, cell size and shape [7]. Chemical cellular lysis is usually performed by using three different kinds of detergents. Ionic detergents such as sodium dodecyl sulphate (SDS) lyse cells in a matter of seconds and tend to denature proteins. Non-ionic detergents, like Triton X-100, lyse cells at a much slower rate and have a much lower tendency to denature proteins. Usage of zwitterionic detergents results in no net change in the charge of solubilized protein; however, they might have downstream effects in electrophoretic separation methodologies which can result in the reversal of electro-osmotic flow [4].

So far, a few microfluidic platforms have been developed for single cell lysis. However, a high throughput method of lysing single mammalian cells has not been reported elsewhere. Confining single cells into individual droplets would be an ideal method to compartmentalize the cell lysate for analysis of intracellular compounds. Droplet-based microfluidics is a promising platform for single cell encapsulations and analysis [8]. The field of droplet based microfluidics has honed important techniques such as single cell assays, drug screening assays [9] and polymerase chain reaction (PCR) $[10,11]$ among others. Continuous lyses of bacterial cells and the fractionation/detection of intracellular proteins had earlier been studied by Schilling et al. [12]. A suspension of E. coli cells $\left(10^{8}\right.$ cells/ $\mathrm{mL}$ ) and Bacterial Protein Extraction Reagent (BPER) was pumped through a three-inlet, two-outlet microfluidic device. Following that, a fluorogenic enzyme assay was used to detect extracted intracellular components ( $\beta$-galactosidase). Further advancements in this area were performed by Novak et al. [11] and Zeng et al. [13] where bacterial cells were encapsulated and lysed within droplets followed by PCR within droplets. Encapsulation of individual cells in the droplets relies on high flow rates for cell ordering in the micro channels. Due to the difficulties of high flow rate fluid controls and challenges faced due to cellular aggregations, lysis of single cells was less reported.

The aim of this study is to develop an integrated droplet based microfluidic platform for single mammalian cell encapsulation and lysis in picolitre droplets. The cells encapsulated in the droplets are lysed using a low concentration of Triton X-100. Individual cell lysis inside the droplets was enhanced by incorporating winding/serpentine channels which contribute to unequal re-circulating flows resulting in asymmetrical fluid vortices inside the droplets [14]. Permeabilization of the eukaryotic cell membrane leading to loss of eukaryotic cell membrane integrity or lysis was validated by using fluorescent staining

*Corresponding author: Chen Chia Hung, Singapore Institute for Neurotechnology (SiNAPSE), Singapore, E-mail: biecch@nus.edu.sg

Received February 18, 2013; Accepted March 06, 2013; Published March 08 2013

Citation: Ramji R, Xiang AC, Ying NJ, Teck LC, Hung CC (2013) Microfluidic Single Mammalian Cell Lysis in Picolitre Droplets. J Biosens Bioelectron S12: 001. doi:10.4172/2155-6210.S12-001

Copyright: $\odot 2013$ Ramji R, et al. This is an open-access article distributed under the terms of the Creative Commons Attribution License, which permits unrestricted use, distribution, and reproduction in any medium, provided the original author and source are credited. 
method. This novel platform would allow us to compartmentalize single cells and further analyze intracellular proteins in a high throughput manner.

\section{Materials and Methods}

The microfluidic device was fabricated with polydimethylsiloxane (PDMS, Sylgard 184, Dow Corning, USA). A silicon master mold containing the microfluidic channels (junction width $30 \mu \mathrm{m}$ and height $50 \mu \mathrm{m})$ was fabricated by photolithography process. Briefly, SU8-2050 photo resist (Micro Chem Co, USA) was coated onto a silicon wafer at $500 \mathrm{rpm}$ for 20 seconds and then at $3500 \mathrm{rpm}$ for 30 seconds. The resist was then soft baked at $65^{\circ} \mathrm{C}$ for 3 minutes and then at $95^{\circ} \mathrm{C}$ for 9 minutes on a hot plate. Next, the wafer was placed together with the photo mask on the mask aligner and exposed under ultraviolet light for 30 seconds. After exposure, the wafer was baked on a hot plate at $65^{\circ} \mathrm{C}$ for 2 minutes and then at $95^{\circ} \mathrm{C}$ for 7 minutes. This wafer was then developed using SU-8 developer (Micro Chem Co, USA) for 15 minutes while being shaken gently. Lastly, the wafer was removed from the SU8 Developer and washed with isopropyl alcohol (IPA) before being blown dry with nitrogen. The master mold was then silanized inside a desiccator for 15 minutes with Trichloro $(1 \mathrm{H}, 1 \mathrm{H}, 2 \mathrm{H}, 2 \mathrm{H}$-perfluorooctyl) silane (Sigma, Singapore). Upon coating with silane, PDMS mixture was poured and cured on the master mold to replicate the microfluidic channels. The PDMS containing the microchannels was then cut and peeled off from the master. Inlet and outlet ports were made by using a $1 \mathrm{~mm}$ and 1.5 mm Harris Uni-Core TM puncher (Ted Pella Inc, USA), respectively. The PDMS master replica was then bonded to a PDMS coated glass slide, using oxygen plasma cleaner (March Instrument Inc., USA). In order to further strengthen the bonding, the PDMS layer was baked overnight in the oven at $70^{\circ} \mathrm{C}$.

A431 cell line obtained from American Type Culture Collection (Manassas, USA) was cultured in a T-75 flask with Dulbecco's Modified Eagle Medium DMEM (HyClone, Thermo Scientific, USA) as growth media. The media was supplemented with $4.5 \mathrm{~g} / \mathrm{L}$ glucose, $4 \mathrm{mM}$ L-glutamine, phenol red (Hyclone, USA), 1 mM Sodium Pyruvate, 10\% FBS (Hyclone, USA), $50000 \mathrm{IU} / \mathrm{L}$ penicillin and $50 \mathrm{mg} / \mathrm{L}$ streptomycin. Cells were grown at $37^{\circ} \mathrm{C}$ in a humidified incubator maintained at $5 \%$ $\mathrm{CO}_{2}$. Once the cells were confluent, they were harvested by a 10 minutes treatment with $2 \mathrm{X}$ 0.5\% Trypsin-EDTA (Caisson Labs, USA) and finally re-suspended in $2 \mathrm{ml}$ of DMEM medium. This suspension was passed through a cell strainer with $40 \mu \mathrm{m}$ pore size (BD Biosciences, Singapore) to filter out cell clusters/aggregates. The filtered cells were centrifuged followed by the removal of DMEM media and were washed twice and re-suspended uniformly in $1 \mathrm{X} \mathrm{pH} 7.2$ phosphate buffered saline solution (Sigma, Singapore). The cells were counted using a standard hemocytometer and a cell density containing $7 \times 10^{6}$ cells $/ \mathrm{mL}$ was used for the experiments.

The lysis buffer was carried out on a 96 well plate (Black well transparent bottom, Greiner) with an A431 cell density of $\approx 30,000$ cells/well. Upon seeding the cells inside the well plate, they were incubated overnight at $37^{\circ} \mathrm{C}$ in order for them to adhere to the bottom surface. Following this, the cell media was removed and the cells were washed twice with 1X PBS (Sigma, Singapore). The lytic reagent, either Triton X-100 (Sigma, Singapore) or Cell Lytic M (Sigma, Singapore) of $100 \mu \mathrm{L}$ volume, was added into each well along with a $4 \mu \mathrm{M}$ ethidium homodimer-1. The rate at which the cells were lysed was determined by measuring the fluorescence readout at Ex-495 nm, Em-635 nm using Fluo Star Optima (BMG Labtech, Singapore).

The cells were mixed with $4 \mu \mathrm{M}$ ethidium homodimer-1, EthD-
1 and $2 \mu \mathrm{M}$ Calcein-AM (Life Technologies, Singapore). The cell mixture was collected on a $1 \mathrm{~mL}$ plastic syringe (BDTM, Singapore). Fluocarbon oil HFE 7500 (3M NovecTM, Singapore) with 2\% krytox (modified) surfactant was used to generate droplets. Reagents for cell lysis such as Cell Lytic M and Triton X-100 (Sigma, Singapore) were used accordingly. All the syringes were connected to their respective cell, oil and reagent inlet ports on the microfluidic device with $1 \mathrm{~mm}$ (OD) tubing and were operated using NE-1000 syringe pumps (New Era Pump Systems Inc., USA). The flow rate of the continuous (oil) phase was $10 \mu \mathrm{L} / \mathrm{min}$ while that for reagent and cell mixture were 2 $\mu \mathrm{L} / \mathrm{min}$ and $5 \mu \mathrm{L} / \mathrm{min}$ respectively. The cell encapsulated droplets were collected in a syringe initially and were later injected into an observation chamber (width-2 mm, height- $80 \mu \mathrm{m}$ ). Droplet generation and cell encapsulation was imaged using Photron SA-3 high speed camera at frame rates of 6000-10000 frames/sec. The droplets were placed on a Nikon TE-2000U fluorescent microscope and were imaged using a colored CCD camera with blue and green filter cubes for the live or dead staining respectively. The fluorescent and bright field images were post-processed using Image J software.

\section{Results and Discussion}

Droplet generation for cell encapsulation was carried out in a PDMS microfluidic device with a rectangular cross section (Figure 1A). Cells flowing through the inlet were allowed to pass through a pinching channel with a $30 \mu \mathrm{m}$ and $60 \mu \mathrm{m}$ contraction and expansion width, respectively. This region helped to focus the cells into a single stream further helping to break any cell aggregates. The cell stream together with a parallel reagent stream containing cell lysis buffer entered the droplet forming junction where the cell solution and the lysis buffer were encapsulated into the droplet. The cell encapsulated droplet consisted of the live or dead stain with cell permeable calcein AM and cell impermeable ethidium homodimer-1 (EthD-1). In order to enhance mixing inside the droplets they were passed through serpentine mixers followed by an incubation chamber (double spiral) and finally were collected into a syringe at the channel outlet. Figure 1B shows the schematic representation of the entire process, i.e., from cell encapsulation to intracellular signal analysis.

A deterministic encapsulation of cells inside the droplets was observed in our experiments with $\approx 52 \%$ of the droplets encapsulating single cells (Figure $2 \mathrm{~A}$ ). Single cell encapsulation efficiency was mainly

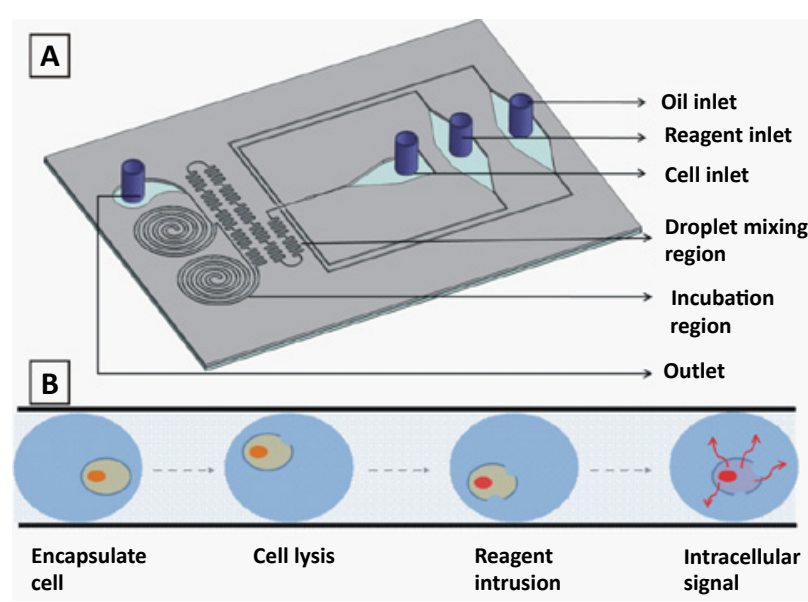

Figure 1: (A) Schematic representation of the microfluidic chip used for single cell lysis; (B) Representation of the individual process i.e., from single cell encapsulation to intracellular signal analysis from the lysed cells. 

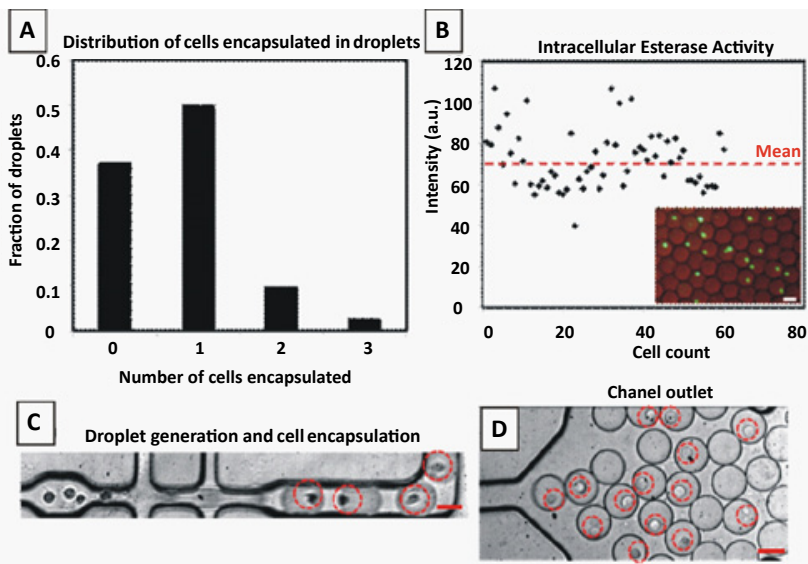

Figure 2: (A) Representation of deterministic encapsulation of single cells into pico-droplets; (B) Plot representing the distribution of intracellular esterase activity seen in cells; the scale bar refers to $50 \mu \mathrm{m} ;(\mathbf{C}, \mathbf{D})$ Images of the droplet forming region and the channel outlet showing single cells encapsulated into individual droplets.
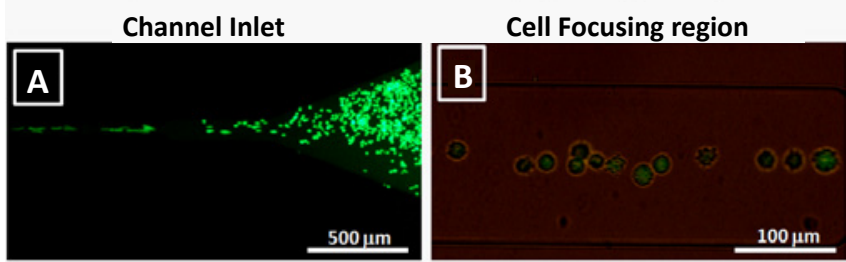

Inside Observation Chamber
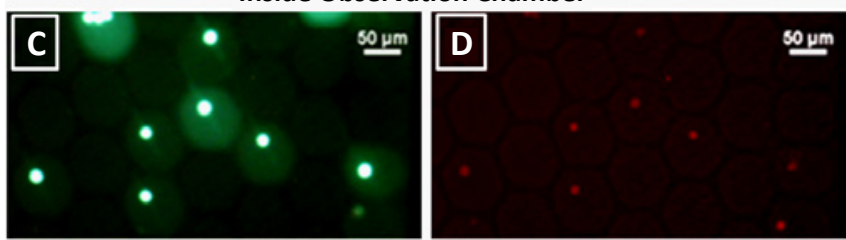

Figure 3: (A) Live cells entering the microchannel stained with calcein AM dye; (B) Cells focused to the centre of the microchannel due to the inertial effects. Droplets collected in the observation chamber viewed with representative (C) green and (D) red emission filter cubes to show live cells whose membrane was just lysed thus allowing EthD-1 to enter inside them.

dependent on the cell density and flow velocity of the oil and aqueous phase. A vertical setup of the syringe pump containing the cells was used in order to overcome cell settling issues and to lower down the non-uniform distribution of the cells at the channel inlet. The encapsulation efficiency can further be improved by using a syringe stirrer to produce a completely homogenous cell distribution arriving at the inlet. This would help in sorting single cells into individual droplet compartments and further help in analyzing their intracellular enzyme activity (Figures 2B-2D). Such single cell expression studies would help us understand cellular heterogeneity.

The cell solution at the inlet was premixed with the live/dead stain i.e., calcein-AM and ethidium homodimer-1 fluorescent dye. Calcein$\mathrm{AM}$ is a substrate that is freely transported through the intact cell membrane of live cells. The intracellular esterases present within the cell catalyze the removal of the acetomethoxy group thus allowing the polyanionic calcein dye to be retained within the cell resulting in an intense fluorescence (Figures $3 \mathrm{~A}$ and $3 \mathrm{~B}$ ) from the calcein dye present within the cell (Ex-495 nm, Em-515 nm). Cell lysis buffer containing
$1 \%$ Triton X-100 (v/v) in 1X Phosphate buffered saline (PBS) was used to lyse the cells. Figures $3 \mathrm{C}$ and $3 \mathrm{D}$ represent an image taken in the observation chamber for calcein AM and ethidium homodimer-1 dyes. Live cells that were just lysed inside the droplets indicated loss of membrane integrity by allowing EthD-1 to bind to the nucleic acids present inside the nucleus.

It was worth to note that the cell lysis reagents used were optimized to lyse individual cells within droplets. Ethidium homodimer-1, which was usually used to report loss of cell membrane integrity, has been applied to validate cell lysis. Upon cell lysis, this membrane impermeable dye freely diffuses into the cytoplasm and binds to the DNA exhibiting a 40 fold enhancement of fluorescence signal (Ex-495 $\mathrm{nm}, \mathrm{Em}-635 \mathrm{~nm}$ ). The lysis buffer used to permeabilize the membrane of A431 cells inside the droplets was initially optimized on cells cultured on a well plate. A commonly used non-denaturing cell lysis reagent i.e., Triton X-100 was compared with that of a commercially available cell lytic-M solution. Intensity plots as seen in figures $4 \mathrm{~A}$ and $4 \mathrm{~B}$ represent the time taken for the cells to die i.e., for the EthD-1 dye to penetrate into the cells and produce fluorescence. Saturation of the curve as represented by the blue arrow marks relate to death of all the cells in the well as a result of which EthD-1 would have bound (saturated) to the all the DNA present in the cells with no more increase in the emitted fluorescence. A concentration of $1 \%$ Triton X-100 was found to lyse the cells faster when compared to the other reagents. Hence, an optimal concentration of $1 \%$ Triton X-100 was used for on chip lysis of cells inside the droplets. Different cell lines, such as the PC9 cells (a lung cancer cell line) were also conducted to the on-chip lysis experiments to confirm the flexibility and reliability of single cell lysis within the droplets.

\section{Conclusions}

An on-chip single cell lysis method has been described in this study. The cells were lysed using Triton X-100, a non-denaturing surfactant. Two important observations validated the lysis of single cells inside the encapsulated droplets. Firstly, the polyanionic calcein dye leaked out of the cell causing an increase in the background fluorescence of the droplet. This would happen only when the cell membrane is permeabilized. Secondly, almost $>98 \%$ of the cell encapsulated droplets exhibited the EthD-1 fluorescence thus reconfirming the lysis of cells allowing the dye to penetrate into the cell and bind to the nucleic acids. This method would facilitate intracellular enzymatic or protein analysis at the single cell level by confining and analyzing single cell lysate within individual compartments. Furthermore, this method would serve as a stepping stone to allow ligand/drug binding studies targeted
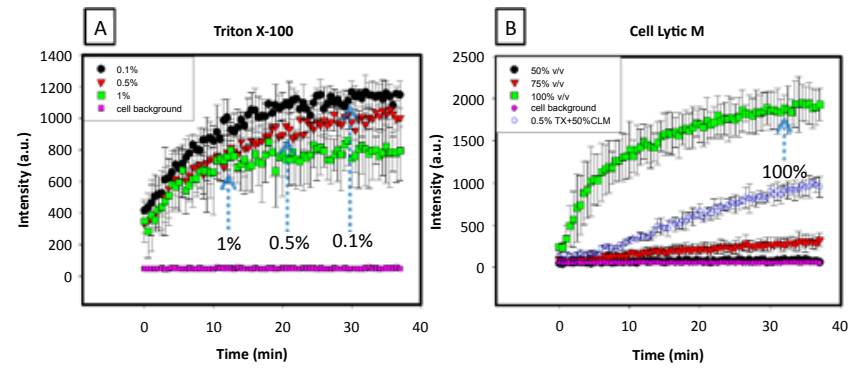

Figure 4: Intensity plots representing cell death in a 96 well plate. (A) Describes the rate of cell death for increasing concentrations $(0.1,0.5,1 \%)$ of Triton X-100; (B) Describes the rate of cell death for increasing concentrations of Cell Lytic M solution. A combined effect of $50 \% \mathrm{v} / \mathrm{v}$ cell lytic and $0.5 \%$ Triton $\mathrm{X}-100$ is also shown. 
Citation: Ramji R, Xiang AC, Ying NJ, Teck LC, Hung CC (2013) Microfluidic Single Mammalian Cell Lysis in Picolitre Droplets. J Biosens Bioelectron S12: 001. doi:10.4172/2155-6210.S12-001

Page 4 of 4

at intracellular compounds and aid in the development of biomarkers for disease diagnosis.

\section{Acknowledgements}

We would like to acknowledge the funding provided by the NUS start-up grant R397000137133 and the SMART research grant R397000146592.

\section{References}

1. Carlo DD, Lee LP (2006) Dynamic Single-Cell Analysis for Quantitative Biology. Anal Chem 78: 7918-7925.

2. Bauer KD, Jacobberger JW (1994) Chapter 23 Analysis of Intracellular Proteins. Methods in Cell Biology 41: 351-376.

3. Krutzik PO, Nolan GP (2003) Intracellular phospho-protein staining techniques for flow cytometry: Monitoring single cell signalling events. Cytometry A 55: 61-70.

4. Brown RB, Audet J (2008) Current techniques for single-cell lysis. J R Soc Interface 5: S131-S138.

5. Sims CE, Meredith GD, Krasieva TB, Berns MW, Tromberg BJ, et al. (1998) Laser-micropipet combination for single-cell analysis. Anal Chem 70: 45704577.

6. Yun SS, Yoon SY, Song MK, Sin-Hyeog Im, Kim S, et al. (2010) Handheld mechanical cell lysis chip with ultra-sharp silicon nano-blade arrays for rapid intracellular protein extraction. Lab Chip 10: 1442-1446.
7. Teissie J (1992) Time course of Electropermeabilization. Charge and field effects in biosystems 3: 285-301.

8. Joensson HN, Andersson SH (2012) Droplet Microfluidics-A Tool for Single-Cell Analysis. Angew Chem Int Ed Engl 51: 2176-12192.

9. Brouzes E, Medkova M, Savenelli N, Marran D, Twardowski M, et al. (2009) Droplet microfluidic technology for single-cell high-throughput screening. Proceedings of the National Academy of Sciences of the USA.

10. Kiss MM, Ortoleva-Donnelly L, Beer NR, Warner J, Christopher G, et al. (2008) High-throughput quantitative polymerase chain reaction in picoliter droplets. Anal Chem 80: 8975-8981.

11. Novak R, Zeng Y, Shuga J, Venugopalan G, Fletcher DA, et al. (2011) SingleCell Multiplex Gene Detection and Sequencing with Microfluidically Generated Agarose Emulsions. Angew Chem Int Ed 50: 390-395.

12. Schilling EA, Kamholz AE, Yager $P$ (2002) Cell lysis and protein extraction in a microfluidic device with detection by a fluorogenic enzyme assay. Anal Chem 74: $1798-1804$.

13. Zeng Y, Novak R, Shuga J, Smith MT, Mathies RA (2010) High-performance single cell genetic analysis using microfluidic emulsion generator arrays. Anal Chem 82: 3183-3190.

14. Bringer MR, Gerdts CJ, Song H, Tice JD, Ismagilov RF (2004) Microfluidic systems for chemical kinetics that rely on chaotic mixing in droplets. Phil Trans R Soc Lond A 362: 1087-1104. 